\title{
The Right-Hand Derivative of an Integral
}

\section{Timothy G. Feeman and Osvaldo Marrero}

In this note, we provide a general result that explains the examples contained in [1], [3], [4], and [5]. We also observe with interest that this problem seems to have been proposed anew at nearly regular intervals of ten to fifteen years. The article [2] also contains a discussion of this problem.

Using the notation in [3], suppose that $f$ is a real-valued function defined on the interval $[0,1]$ such that (a) $f$ is bounded on $[0,1]$, (b) $f$ is continuous on $(0,1]$, and (c) $\lim _{x \rightarrow 0^{+}} f(x)$ does not exist. Then let $F(x)=\int_{0}^{x} f(t) d t$ for $x$ in $[0,1]$. The question is whether or not the right-hand derivative

$$
F^{\prime}+(0)=\lim _{x \rightarrow 0^{+}} \frac{\int_{0}^{x} f(t) d t}{x}
$$

exists.

Before we state our general result, let $\phi$ be a twice differentiable, decreasing, nonnegative function defined on the interval $(0,1]$ such that $\phi^{\prime}(x)$ is increasing on $(0,1]$, $\lim _{x \rightarrow 0^{+}} \phi(x)=\infty$, and $\lim _{x \rightarrow 0^{+}} x \phi^{\prime}(x)=-\infty$. Next, let $g$ be a real-valued function defined on $[0, \infty)$ that is continuous and bounded on $[0, \infty)$, has a bounded antiderivative $G$ on $[0, \infty)$, and has the property that $\lim _{x \rightarrow \infty} g(x)$ does not exist.

Theorem 1. With $\phi, g$, and $G$ as just described, let $f(x)=g(\phi(x))$. Then

$$
\lim _{x \rightarrow 0^{+}} x^{-1} \int_{0}^{x} f(t) d t=0 .
$$

Proof. Suppose that $|G(x)| \leq M$ for all $x \geq 0$. Note that the conditions on $\phi$ imply that $\lim _{x \rightarrow 0^{+}} \phi^{\prime}(x)=-\infty$. Thus, $\lim _{t \rightarrow 0^{+}} G(\phi(t)) / \phi^{\prime}(t)=0$.

Letting $u=1 / \phi^{\prime}$ and $v=G \circ \phi$ and integrating by parts, we have

$$
\int_{0}^{x} f(t) d t=\int_{0}^{x} g(\phi(t)) d t=\frac{G(\phi(x))}{\phi^{\prime}(x)}-\int_{0}^{x} G(\phi(t))\left(\frac{1}{\phi^{\prime}(t)}\right)^{\prime} d t .
$$

Hence,

$$
\begin{aligned}
\left|\int_{0}^{x} f(t) d t\right| & \leq \frac{M}{\left|\phi^{\prime}(x)\right|}+M \int_{0}^{x}\left|\left(\frac{1}{\phi^{\prime}(t)}\right)^{\prime}\right| d t \\
& =\frac{M}{\left|\phi^{\prime}(x)\right|}-M \int_{0}^{x}\left(\frac{1}{\phi^{\prime}(t)}\right)^{\prime} d t \\
& =\frac{M}{\left|\phi^{\prime}(x)\right|}+\frac{M}{\left|\phi^{\prime}(x)\right|} \\
& =\frac{2 M}{\left|\phi^{\prime}(x)\right|} .
\end{aligned}
$$


Dividing by $x$ yields

$$
\left|\frac{\int_{0}^{x} f(t) d t}{x}\right| \leq \frac{2 M}{\left|x \phi^{\prime}(x)\right|} .
$$

From the hypothesis that $\lim _{x \rightarrow 0^{+}} x \phi^{\prime}(x)=-\infty$, it now follows that

$$
\lim _{x \rightarrow 0^{+}} x^{-1} \int_{0}^{x} f(t) d t=0 .
$$

This completes the proof.

For example, taking $g(x)=\sin x$ and $\phi(x)=1 / x$ gives the result in [1] and [4], while $g(x)=\cos x$ and $\phi(x)=1 / x$ are used in [5].

If $\phi(x)=-\ln x$, then the hypothesis that $\lim _{x \rightarrow 0^{+}} x \phi^{\prime}(x)=-\infty$ is not met. The main result of [3] is that $\lim _{x \rightarrow 0^{+}} x^{-1} \int_{0}^{x} \sin (\ln t) d t$ does not exist. We now take a closer look at this phenomenon and produce a family of functions $\phi_{n}$ such that $\lim _{x \rightarrow 0^{+}} x^{-1} \int_{0}^{x} \sin \left(\phi_{n}(t)\right) d t$ does not exist for any $n$.

Specifically, let $\phi_{1}(t)=\ln t$ for $t>0$ and, for each natural number $n$, define $\phi_{n+1}$ by the recursive formula $\phi_{n+1}(t)=\ln \left(\ln \left(e^{\phi_{n}(t)}+1\right)\right)$ for $t>0$. For instance, $\phi_{2}(t)=$ $\ln (\ln (t+1))$ and $\phi_{3}(t)=\ln (\ln (\ln (t+1)+1))$. We note that $\lim _{t \rightarrow 0^{+}} \phi_{n}(t)=-\infty$ and also that $\lim _{t \rightarrow 0^{+}} t \phi_{n}{ }^{\prime}(t)=1$ for all $n \geq 1$. Two less-obvious facts are recorded in the following lemmas.

Lemma 1. For the functions $\phi_{n}$ it is the case that $\lim _{t \rightarrow 0^{+}} e^{\phi_{n}(t)} \cdot \phi_{n}{ }^{\prime}(t)=1$ for all $n \geq 1$.

Proof. We proceed by induction, noting that the result is clear when $n=1$, in which case $e^{\phi_{1}(t)} \phi_{1}^{\prime}(t)=1$ for all $t>0$.

For the inductive step, the relation $\phi_{n+1}(t)=\ln \left(\ln \left(e^{\phi_{n}(t)}+1\right)\right)$ implies that

$$
\begin{aligned}
\phi_{n+1}{ }^{\prime}(t) & =\frac{1}{\ln \left(e^{\phi_{n}(t)}+1\right)} \cdot \frac{d}{d t}\left(\ln \left(e^{\phi_{n}(t)}+1\right)\right) \\
& =\frac{1}{e^{\phi_{n+1}(t)}} \cdot \frac{1}{e^{\phi_{n}(t)}+1} \cdot \frac{d}{d t}\left(e^{\phi_{n}(t)}+1\right) \\
& =\frac{1}{e^{\phi_{n+1}(t)}} \cdot \frac{1}{e^{\phi_{n}(t)}+1} \cdot e^{\phi_{n}(t)} \cdot \phi_{n}{ }^{\prime}(t) .
\end{aligned}
$$

Multiplying both sides of this by $e^{\phi_{n+1}(t)}$, we obtain

$$
e^{\phi_{n+1}(t)} \cdot \phi_{n+1}{ }^{\prime}(t)=\frac{1}{e^{\phi_{n}(t)}+1} \cdot e^{\phi_{n}(t)} \cdot \phi_{n}{ }^{\prime}(t) .
$$

In the limit as $t$ tends to 0 from the right, the first factor on the right tends to 1 . Hence,

$$
\lim _{t \rightarrow 0^{+}} e^{\phi_{n+1}(t)} \cdot \phi_{n+1}{ }^{\prime}(t)=\lim _{t \rightarrow 0^{+}} e^{\phi_{n}(t)} \cdot \phi_{n}{ }^{\prime}(t)=1
$$

by the induction hypothesis. This completes the proof of the lemma.

Lemma 2. For all $n \geq 2, \lim _{t \rightarrow 0^{+}}\left[\phi_{n}(t)-\ln t\right]=0$. 
Proof. Fix a natural number $n \geq 2$. The expression $\lim _{t \rightarrow 0^{+}} e^{\left[\phi_{n}(t)-\ln t\right]}$ or, what is the same thing, $\lim _{t \rightarrow 0^{+}} e^{\phi_{n}(t)} / t$ is an indeterminate form. Applying L'Hôpital's rule and Lemma 1, we see that $\lim _{t \rightarrow 0^{+}} e^{\phi_{n}(t)} \cdot \phi_{n}{ }^{\prime}(t)=1$. Thus, $\lim _{t \rightarrow 0^{+}} e^{\left[\phi_{n}(t)-\ln t\right]}=1$ as well. Taking natural logarithms of both sides completes the proof.

We are finally ready to establish the claim we originally made about the functions $\phi_{n}$.

Theorem 2. For every natural number $n$, the limit $\lim _{x \rightarrow 0^{+}} x^{-1} \int_{0}^{x} \sin \left(\phi_{n}(t)\right) d t$ does not exist.

Proof. As already mentioned, the case where $n=1$ is the main result of [3]. Indeed, integration by parts, applied twice, shows that $\int_{0}^{x} \sin (\ln t) d t=(x / 2)[\sin (\ln x)-$ $\cos (\ln x)]$. It follows that, as $x \rightarrow 0^{+}$, the value of $x^{-1} \int_{0}^{x} \sin (\ln t) d t$ oscillates between the values $\pm 1 / \sqrt{2}$.

For the remaining cases, fix a natural number $n \geq 2$ and let $\epsilon>0$ be an arbitrary positive number. The sine function is uniformly continuous on the whole real line, so there exists a positive number $\delta>0$ such that $|\sin x-\sin y|<\epsilon$ whenever the real numbers $x$ and $y$ satisfy $|x-y|<\delta$. By Lemma 2, there exists a positive number $b>0$ such that $\left|\ln t-\phi_{n}(t)\right|<\delta$ whenever $0<t \leq b$. Thus, for such $t$, we have $\left|\sin (\ln t)-\sin \left(\phi_{n}(t)\right)\right|<\epsilon$. We infer that, for $0<x \leq b$,

$$
\begin{aligned}
\left|\frac{\int_{0}^{x} \sin (\ln t) d t}{x}-\frac{\int_{0}^{x} \sin \left(\phi_{n}(t)\right) d t}{x}\right| & \leq \frac{\int_{0}^{x}\left|\sin (\ln t)-\sin \left(\phi_{n}(t)\right)\right| d t}{x} \\
& <\frac{\int_{0}^{x} \epsilon d t}{x} \\
& =\epsilon .
\end{aligned}
$$

Since $\epsilon>0$ was arbitrary and the value of $x^{-1} \int_{0}^{x} \sin (\ln t) d t$ oscillates between $\pm 1 / \sqrt{2}$, the theorem follows.

The proof of Theorem 2 suggests that the value of $x^{-1} \int_{0}^{x} \sin \left(\phi_{n}(t)\right) d t$ might oscillate between the values $\pm 1 / \sqrt{2}$ for all $n$ as $x \rightarrow 0^{+}$, a suggestion that is supported by some numerical estimates we have made using Maple. It is also apparent from the proof that the sine function could be replaced by any function $g$ that is uniformly con-

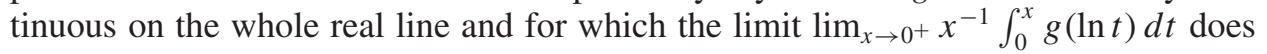
not exist. Apart from the cosine function (an obvious alternative), we are not aware of any other such function.

In closing, we thank the referee for pointing out that the authors of [2] have shown that, for a function $\phi$ that is continuously differentiable on $(0,1]$, has a monotone derivative, and satisfies $\lim _{t \rightarrow 0^{+}} \phi(t)= \pm \infty$, the additional condition of $\lim _{t \rightarrow 0^{+}} t \phi^{\prime}(t)$ being finite guarantees that the right-hand derivative

$$
\lim _{x \rightarrow 0^{+}} x^{-1} \int_{0}^{x} \sin (\phi(t)) d t
$$

does not exist. The functions $\phi_{n}$ introduced in the preceding illustrate this basic fact. 
1. R. E. Dowds and R. J. Driscoll, Solution to proposed problem E 1970, Amer. Math. Monthly 75 (1968) 678.

2. P. Fishback, N. Ceglarek, and T. Moleski, On the differentiability of $\int_{0}^{x} \sin (1 / t) d t$ and $\int_{0}^{x} \sin (\ln (t)) d t$, Math. Mag. 70 (1997) 218-222.

3. John Klippert, On the right-hand derivative of a certain integral function, Amer. Math. Monthly 98 (1991) 751-752.

4. S. Ricci, Solution to proposed problem 1112, Math. Mag. 55 (1982) 48.

5. A. Wilansky and G. Piranian, Solution to proposed problem E 1071, Amer. Math. Monthly 61 (1954) 124.

Villanova University, Villanova, PA 19085-1699

timothy.feeman@villanova.edu

osvaldo.marrero@villanova.edu 